\title{
PUERTO VALLARTA: ESPACIO TURÍSTICO LGBT CONFIGURADO A TRAVÉS DE RELACIONES DE PODER EN RED
}

\author{
Jesús OtONIEL SOSA RODRíGUeZ \\ otonielsosa@ucol.mx \\ Universidad de Colima
}

Los espacios turísticos se configuran por prácticas sociales y relaciones de poder sostenidos por los diferentes actores locales que participan en el fenómeno del turismo, influidos por el contexto nacional e internacional. El objetivo de este artículo es analizar el proceso de configuración del espacio turístico LGBT+ en Puerto Vallarta, Jalisco, a través de las relaciones de poder en red que sostienen los diferentes actores involucrados en el destino. Este estudio se realizó a través de la etnografía como método cualitativo y el análisis de redes sociales (ARS) como método cuantitativo. Los resultados demuestran la creación de una red de actores con relaciones de poder asimétricas que han influido en configurar parte del destino turístico en un espacio amigable con la comunidad LGBT+.

Palabras clave: Turismo LGBT+, relaciones de poder, etnografía, espacio turístico, Puerto Vallarta.

\section{PUERTO VALLARTA: LGBT TOURISTIC SPACE CONFIGURED THROUGH NETWORK POWER RELATIONS}

Touristic spaces are configured by social practices and power relations sustained by the different local actors involved in the phenomenon of tourism, influenced by the national and international context. The objective of this article is to analyze the process of configuration of LGBT+ touristic space in Puerto Vallarta, Jalisco, through the networked power relations that sustain the different actors involved in the destination. This study was conducted through ethnography as a qualitative method and social network analysis (ARS) as a quantitative method. The results demonstrate the creation of a network of actors with asymmetrical power relations that have influenced to configure part of the touristic destination in a space friendly to the LGBT+ community.

Keywords: LGBT+ Tourism, power relations, ethnography, touristic space, Puerto Vallarta. 


\section{Introducción}

a constitución de un espacio turístico LGBT+ ${ }^{1}$ en Puerto Vallarta, Jalisco, México forma parte de un proceso socio-histórico en el que han participado diversos actores, cuyas acciones se desenvuelven en un contexto de condiciones y relaciones de poder cambiantes a lo largo del tiempo.

Puerto Vallarta se transformó de pequeño pueblo pesquero y salinero a ser un enclave turístico de relevancia nacional e internacional, convertido en un espacio socio-territorial que permite prácticas sociales a la comunidad $\mathrm{LGBT}+{ }^{2}$, de forma particular a los gais ${ }^{3}$, que en otros contextos nacionales e internacionales no les son permitidas. Esos cambios son el resultado de la resistencia de la misma comunidad a la diversidad sexual, que se ha organizado y hace frente a través de diferentes estrategias que coadyuvan actores: turistas, residentes, empresarios, asociaciones y gobierno. La suma y diferenciación de los capitales de los diversos actores han (re) configurado las relaciones de poder y estas a su vez transforman la zona romántica, también conocida como Viejo Vallarta y Zona Sur, así como Amapas, que se han convertido en ese espacio turístico diverso que insiste en crecer y consolidarse.

El objetivo de este trabajo es analizar las relaciones de poder económicas, sociales y políticas que entrelazan los diferentes actores participantes en la configuración de Puerto Vallarta como un destino turístico LGBT+. En el análisis se considera que esas relaciones de poder se sostienen por una red entre los distintos actores locales, con un contexto nacional-global más complejo que incide en la configuración socio-territorial de Puerto Vallarta.

Para dar cumplimiento al objetivo señalado, este trabajo se estructura en seis secciones: en primer lugar, se realiza una breve introducción al tema; posteriormente, se presenta el encuadre teórico de la investigación sobre relaciones de poder en red. Enseguida, se desarrolla la metodologíautilizada, para continuar con el contexto que ha configurado a Puerto Vallarta como destino LGTB friendly. En la siguiente sección se muestran los resultados y la discusión sobre las redes sociales y relaciones de poder; como cierre se encuentran las conclusiones derivadas de este trabajo.

\footnotetext{
1 Término que se usa para nombrar a Lesbianas, Gais, Bisexuales y Transexuales.

2 Término que usa el autor para nombrar a la comunidad diversa, mismo que agrega el signo + , con el fin de no dejar fuera a nadie en esta nominación.

3 Se usará el plural "Gais" promovido por la Real Academia Española como el correcto en castellano.
} 


\section{Relaciones de poder en red: actores y capitales que configuran espacios turísticos}

Las relaciones e interacciones establecidas por los actores transforman los espacios urbanos en territorios apropiados a través del tiempo. Así, aunque la globalización ha permitido formular teorías acerca de la desterritorialización, como la de la creación de una aldea global que contiene a todos los territorios y permite la deslocalización de los recursos, siguiendo a Giménez (2005) los territorios siguen fungiendo como espacios estratégicos en los cuales se desarrollan actividades simbólicas que permiten inscribir esas excepciones culturales, pues mantienen un rol preponderante en términos económicos y políticos.

No basta con entender el territorio desde lo global, sino en conjunto con las prácticas y relaciones de poder de los actores en el espacio local (Giménez, 2007; Daniilidis y Beaumont, 2015; Saarinen y Wall-Reinius, 2019), preguntándose por las características que las constituyen, así como por los procesos y dinámicas de construcción y apropiación que configuran el territorio (Sosa, 2012).

En la época contemporánea, las ciudades experimentan procesos donde queda claro que el tipo de ciudad que se quiere "no puede estar divorciada de la que plantea qué tipo de lazos sociales, de relaciones con la naturaleza, de estilos de vida, de tecnologías y de valores estéticos que se desean" (Harvey, 2008, p. 23). Esa mediación del tipo de ciudad querida o requerida por los habitantes no siempre es decisión de estos, sino del capitalismo que busca colocar excedentes de capital en diferentes proyectos, dos de ellos son ciudad y turismo (Harvey, 2008; Borja, 2013; Benach, 2016).

La ciudad se presenta como un espacio social, en el cual se "recorta" un territorio en el que existen un conjunto de relaciones desiguales entre los actores que ocupan posiciones diferentes dependiendo de la dimensión en la que se encuentren (Bourdieu, 1984; 1996; 1997), es decir, el espacio social construido con base en principios de diferenciación y distribución, dependiendo de los capitales (económico, cultural, simbólico y social) con los que cuenta cada actor.

La suma de capitales de cada actor le sirve para posicionarse en el espacio social establecido, su legitimación resulta de que "los agentes aplican a las estructuras objetivas del mundo social estructuras de percepción y de apreciación que salen de esas estructuras objetivas... esas relaciones objetivas de poder tienden a reproducirse en las relaciones de poder simbólico" (Bourdieu, 1988, p. 138). 
El poder entendido como capacidad relacional es un elemento que dota de importancia a las relaciones sociales, pues a través de ellas y sus discursos se construyen significados respecto de los espacios, las prácticas y roles de los actores permitidos en un enclave turístico (Daniilidis y Beaumont, 2015; Saarinen y WaII-Reinius, 2019).

La sociedad como estructura social surge de las relaciones de conflicto, las cuales se subsanan a través de acuerdos y negociaciones entre los actores que intervienen en la toma de decisiones o que influyen en el ordenamiento social. Son esos mismos actores los que edifican las instituciones ordenadoras de la sociedad y que a su vez tienen la capacidad de agencia para participar y promover los cambios que consideren necesarios en la dinámica social, a fin de mover esa misma estructura (Bronfman, 2000; Giddens, 2006; Castells, 2009).

Para entender ese entramado de relaciones de poder en los campos económico, político y cultural que intervienen en el desarrollo y configuración de un espacio turístico que plantea a su vez resistencia al orden social heteronormativo y dicotómico, se considera la teoría de las redes sociales, entendidas como: "... complejas estructuras de comunicación establecidas en torno a un conjunto de objetivos que garantizan, al mismo tiempo, unidad de propósitos y flexibilidad en su ejecución gracias a su capacidad para adaptarse al entorno operativo" (Castells, 2009, p. 46).

Aunque "las instituciones pueden mantener relaciones de poder que se basan en la dominación que ejercen sobre sus sujetos" (Castells, 2009, p. 33), también se asume que esos sujetos con su capacidad de agencia y situados en posiciones distintas en la red poseen poder, mismo que ejercen para modificar o habilitar el constreñimiento que causan dichas instituciones (Giddens, 2006, Castells, 2009; Daniilidis y Beaumont, 2015; Saarinen y Wall-Reinius, 2019).

Cabe mencionar que el poder no es un atributo, sino una relación asimétrica en donde la influencia de un actor sobre otro para tomar decisiones siempre tendrá la posibilidad de enfrentar resistencias que pongan en duda las relaciones de poder; sin embargo, el proceso de legitimación por el que los valores y las reglas se aceptan por parte de los actores determinará asimismo el proceso de reproducción de las relaciones de poder, así como en las formas de organizarse y tomar decisiones en lo local (Castells, 2009; Saarinen y Wall-Reinius, 2019). 


\section{Metodología}

El diseño de la metodología está enmarcado en el paradigma del constructivismo, con un marco que permite dar respuesta a la pregunta de investigación: ¿cómo las relaciones que existen entre los actores que participan en el fenómeno social del turismo configuran actualmente un espacio turístico LGBT+ friendly en Puerto Vallarta?

Para dar respuesta, se decidió que el método mixto era el más adecuado en esta investigación pues más allá de la complementariedad que el método cualitativo y cuantitativo puedan tener (Tashakkori y Teddlie, 1998; Cortés et al., 2008), resulta conveniente el uso mixto, dado que el método cuantitativo tiene técnicas que permiten acercarse a la explicación de la realidad a través de la construcción y análisis de datos numéricos, mientras que el método cualitativo presenta explicaciones que generan conocimiento no considerado previamente por la teoría; en otras palabras, otra(s) forma (s) de explicar las realidades complejas captando y (re)construyendo su significado.

En esta investigación primero se trabaja el análisis cualitativo de la etnografía en la zona romántica y Amapas de Puerto Vallarta, el cual se complementa con datos cuantitativos para fortalecer algunos argumentos de índole relacional en tanto a relaciones de poder del destino. El método cualitativo a través de la etnografía brinda el marco para explicar la(s) realidad(es) que se están estudiando, al permitir dar cuenta a través de las narrativas de los actores, de la observación participante y la investigación documental in situ cómo es que se piensa y construye un espacio turístico conformado para y por una comunidad LGBT+, recientemente visibilizada por el gobierno federal en tanto a turistas, que ha sido incluida y excluida en los destinos turísticos y cómo es que se están modificando las relaciones sociales en la vida cotidiana, que no se limitan a lo establecido en el orden socio-sexual defendido en el orden heteronormativo.

El proceso cuantitativo se realizó a través del Análisis de Redes Sociales (ARS), método matemático que se basa en el hipercubo, representado de varias maneras: como secuencias binarias $y$, de forma equivalente, como productos cartesianos (Hage y Harary, 2002). Su análisis se usa para determinar las relaciones entre entidades: personas (nombres, correos electrónicos, alias), grupos de personas (relaciones sociales), organizaciones, entre otros. En esta investigación se aplicó este método para codificar las relaciones sociales entre los actores del espacio turístico LGBT+ friendly en Puerto Vallarta. 
Por medio de esta perspectiva híbrida se abordan fenómenos complejos como la configuración de espacios turísticos, lo cual implicó revisar las relaciones de poder (culturales, económicas y políticas) colocadas en procesos de disputa por el orden social, que son atravesados por las relaciones de poder.

Finalmente, la etnografía permitió desarrollar el proceso de teorización en conjunto con el de investigación, es decir, con base en la observación realizada se han propuesto conceptos o explicaciones de la realidad estudiada en campo, tomando en cuenta la propuesta de Guber (2015, p. 32): "mantener un delicado equilibrio entre observar y participar".

\section{El contexto en las relaciones de poder que ha configurado a Puerto Vallarta como un destino LGBT+ friendly}

En el caso de Puerto Vallarta, como en el de otras ciudades del mundo, el poder es un proceso fundamental de su sociedad que se ha definido en un contexto de valores e instituciones pilares del modelo sexual occidental, en donde lo que se ha valorado e institucionalizado ha sido definido por las relaciones de poder (Castells, 2009). De acuerdo con Collignon (2011) el referido modelo sexual occidental dominante se ha caracterizado por una relación sexo-afectiva establecida entre un hombre y una mujer (heterosexualidad), que se formaliza mediante un contrato social (matrimonio) con el fin de reproducción y con límites monogámicos.

Este modelo sexual predominante hasta el siglo XXI ha presentado acciones de resistencia de lo que se ha denominado como "diversidad sexual", concepto "compuesto acuñado durante el siglo XXI que defiende" prácticas que van en contra de la "normalidad sexual". Sin embargo, en este destino turístico el modelo sexual predominante ha sido cuestionado, particularmente en la zona romántica y de Amapas; la multiculturalidad ha permeado en la intersubjetividad de sus residentes y turistas y ha permitido establecer un proceso de resistencia contra dicho modelo, mediante prácticas sexuales como esquemas de resistencia en el sentido político y social. En Puerto Vallarta, se ha abierto un espacio de resistencia a favor de la diversidad sexual, particularmente para los gais, bajo la sombrilla de la actividad económica predominante: el turismo.

Los diversos actores que se analizan en este artículo, están inmersos en estas relaciones de poder, las cuales son "atacadas" por las formas de poder ejercidas en la 
vida cotidiana de turistas y residentes LGBT+ que inicialmente son categorizados e identificados por sus preferencias sexo-genéricas, "atándolos a una ley de verdad que deben reconocer y que los otros deben reconocer en ellos, es una forma de poder que transforma a los individuos en sujetos... sometidos a través del control y la dependencia y sujeto a su propia identidad" (Foucault, 1988, p. 7).

La lucha predominante en la zona romántica de Puerto Vallarta es por el respeto de los derechos humanos de la comunidad diversa, derechos que se ven vulnerados fuera de espacios turísticos y cuya lucha toma como bastión territorial un espacio multicultural (mexicanos y extranjeros, particularmente estadounidenses y canadienses) que buscan combatir la sumisión de la subjetividad de una comunidad vulnerada por su orientación sexo-genérica.

Al iniciar este análisis de las relaciones de poder que han posibilitado la configuración de la zona romántica y de Amapas como un espacio turístico que "permite" el turismo LGBT+, es necesario establecer tres elementos en las relaciones de poder: 1) las relaciones de poder se encuentran arraigadas en el tejido social vallartense, pero bajo un contexto global más amplio que sirve de paraguas en las acciones de los actores; 2) las relaciones de poder son complejas y no son únicamente entre la resistencia (comunidad diversa o diversidad sexual) y el gobierno como garante del Estado, sino que también existen luchas al interior de la misma comunidad LGBT+ y 3) existen distintos dispositivos de poder que coadyuvan al ejercicio del mismo en un contexto socio-espacial enmarcado en relaciones del turismo.

\subsection{El contexto global que favorece la constitución de destinos turísticos LGBT}

La postura de la Organización Mundial del Turismo (OMT) respecto al turismo LGBT+ está respaldada en el ejercicio de los derechos humanos alrededor del mundo y bajo esquemas de destinos, espacios o productos turísticos específicos en los diferentes países que apoyan y reconocen a la comunidad LGBT+. El secretario general de la OMT, Taleb Rifai, declaró en 2017 que "Ios impactos positivos del turismo LGBT+ van más allá de los beneficios económicos. Los destinos que le dan la bienvenida al turismo LGBT+ convergen en una imagen poderosa de tolerancia y respeto... y consecuentemente se convierten en defensores globales de los derechos humanos" (OMT, 2017, p. 8). 
La relación de poder e influencia del reporte está respaldada por la Asociación Internacional de Viajes para Gais y Lesbianas (IGLTA, 2017), así como por la Organización de las Naciones Unidas, todas apoyando la postura de "libertad" de prácticas turísticas, independientemente de la orientación sexual de los individuos. Otros dos referentes a nivel internacional son las empresas de consultorías en turismo LGBT+, Out Now Global que es una empresa en mercadotecnia dirigida a la comunidad gay y lésbica, establecida en Australia desde 1992 y con oficinas en Amsterdam desde 2001. Se consideran "los más experimentados mercadólogos para la comunidad gay y lésbica" porque creen que no hay tal cosa como un gay o lesbiana "típica", sino que la "diversidad" es la clave para entender a este"mercado" (Out Now Global, 2019).

Aunada a esta empresa, una segunda y no menos importante organización que sostiene a través de estudios mercadológicos la importancia del mercado LGBT+ es la Community Marketing \& Insights (CMI), establecida también en 1992, en la capital mundial de la comunidad: San Francisco, California. Esta instancia, además de dedicarse a atender clientes para campañas mercadotécnicas LGBT+, realiza dos conferencias anuales: un simposio sobre mercadotecnia y publicidad LGBTQ y el Foro de Turismo LGBTQ.

Estos cinco actores: ONU, OMT, IGLTA, Out Now y CMI convergen en alentar el turismo LGBT+ mediante una estrategia de reportes e investigaciones que sostienen la importancia económica de la comunidad LGBT+ y también la relevancia de impulsar el turismo para que, a través de este, las prácticas permeen en mayor respeto de los derechos humanos en el mundo. Las relaciones de poder a nivel internacional son más complejas que este nivel presentado; sin embargo, se identifican los actores internacionales más relevantes en el apoyo de los derechos humanos del turismo LGBT+ a nivel global.

A nivel nacional, el principal actor que apoya la configuración de destinos turísticos LGBT+ es el Gobierno Federal, a través de la Secretaría de Turismo (Sectur), respaldados por la Cámara de Senadores durante el sexenio 2012-2018 (Senado de la República, 2014, p. 3). El sector gubernamental es apoyado por diversos empresarios como Talento Consultores, empresa que respalda la certificación de negocios LGBT+ friendly y la Federación Mexicana de Empresarios LGBT+ (Secretaría de Turismo, 2016, 2017a; 2017b y 2018).

Las acciones emprendidas por el Gobierno Federal fueron detonantes de una visibilización mayor de los destinos LGBT+ en México, a partir de 2015, tomando como 
excusa la derrama económica que generaba dicho segmento en el sector turístico. La estrategia no tardó en manifestar detractores que estaban a favor de "la familia tradicional" y de las prácticas de sexualidad dominantes en occidente, a través de la heterosexualidad.

Destinos como Puerto Vallarta, que habían sido gestados con la bandera gay como insignia a favor de la diversidad sexual, tuvieron mayores posibilidades de exigir el respeto de los derechos humanos de su comunidad local y de los visitantes que llegan al territorio constituido a favor de la libre expresión de prácticas entre personas de la comunidad LGBT+.

En el ámbito del estado de Jalisco, un actor que ha aparecido como garante de los derechos humanos para la comunidad LGBT+ en la comunidad vallartense es la Comisión Estatal de Derechos Humanos Jalisco (CEDHJ), que firmó un acuerdo con el Colectivo Vallarta LGBT en octubre de 2018 (Beltrán, 2018), cabe hacer mención que esta alianza sigue vigente y ha conseguido capacitar a los principales actores de la comunidad LGBT+ respecto a la diversidad sexual y los derechos humanos, así como el establecimiento de una "Recomendación general al municipio de Puerto Vallarta respecto a la reasignación para la concordancia sexo-genérica" en favor de la comunidad trans. Como se observa, lo que sucede en esta localidad tiene lugar en un contexto donde poderosos actores nacionales e internacionales han impulsado una agenda de reconocimiento y respeto a ese segmento de turismo.

\subsection{Contexto local y actores en juego}

La manifestación socio-espacial de los procesos enunciados, puede ser estudiada a distintas escalas. En este caso, el área de estudio es un polígono de la ciudad de Puerto Vallarta que abarca una parte central que se ha consolidado como una zona de usos mixtos en la que tanto la oferta habitacional como la de servicios turísticos convencionales o alternativos son definidos como gay friendly, lo que contribuye a dar a esta parte de la ciudad cierto reconocimiento social nacional e internacional (ver figura 1). 
Figura 1. Localización de la zona romántica LGTB + friendly de Puerto Vallarta y principales servicios turísticos
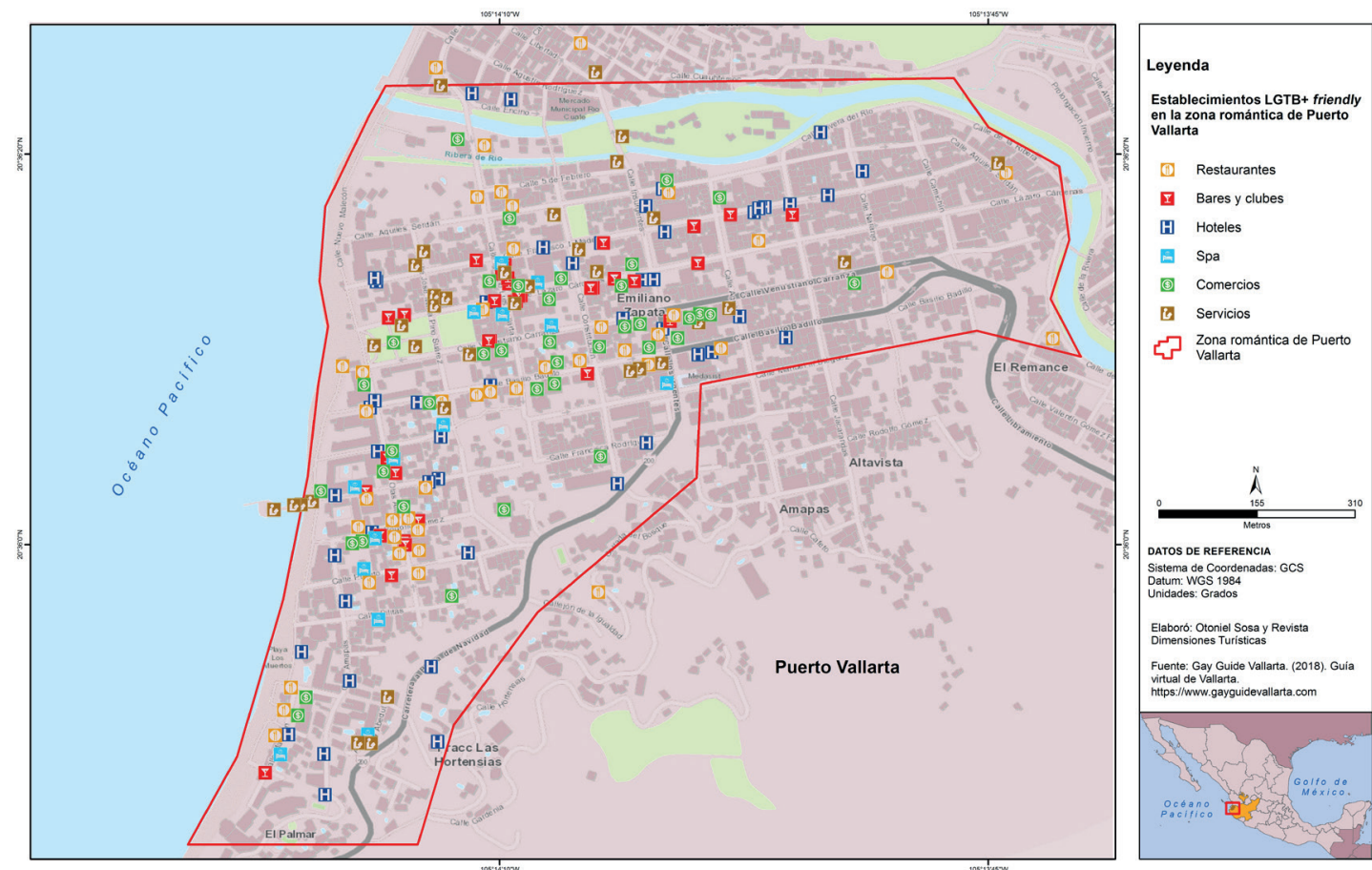

Fuente: Ver interior figura 1.

Las relaciones de poder en el contexto turístico vallartense son tan amplias y complejas como el tejido social mismo de la comunidad. Por ello, en este trabajo se analiza cómo diversos actores utilizan estrategias con dos fines específicos: a) promover el destino como un espacio turístico LGBT+ friendly y b) promover el respeto y defensa de los derechos humanos de la diversidad sexual en Puerto Vallarta, incluyendo a turistas y residentes.

Los esfuerzos establecidos a través de diversas estrategias de enfrentamiento dan lugar a prácticas de resistencia de los actores vallartenses, mismas que han favorecido la configuración de un espacio para la diversidad sexual. En ese sentido esta investigación aporta evidencias empíricas a la tesis que propone que las estrategias usadas por diversos actores sociales tienen lugar en un tejido complejo de relaciones de poder, que moldean la constitución de dinámicas socio-espaciales y contribuyen a definir identidades urbanas y posibilidades económicas para distintos grupos (Foucault,1988; Castells, 2009; Daniilidis y Beaumont, 2015; Saarinen y Wall-Reinius, 2019). En este orden de ideas, destacan a nivel local las acciones 
realizadas por cuatro actores clave, cuyas acciones y dinámicas de interacción han contribuido a configurar el perfil social y económico de la zona estudiada.

a) Gobierno municipal de Puerto Vallarta

Iniciar este análisis con el actor Gobierno no es fortuito, "el ejercicio del poder consiste en conducir conductas y en arreglar las probabilidades" (Foucault, 1988 , p. 15), de tal forma que el caso vallartense no es la excepción; aunque las relaciones de poder no tienen el centro en el gobierno municipal de Puerto Vallarta, este sí cubre las formas instituidas y legítimas de vigilancia y control económico y político en el espacio turístico analizado. Gobierno en el sentido foucaultiano es analizado en tanto un actor que busca estructurar, a través del control y/o vigilancia, el campo de acción de los otros.

b) Amapas Neighborhood Association (ANA)

Esta asociación de vecinos y empresarios, en su mayoría extranjeros, fue creada en 2002 con la intención de atender problemas relativos a la construcción de edificios y la inseguridad del barrio de Amapas, mismo que comparte espacios en la denominada zona romántica y la Playa de los Muertos, que es considerada como la playa gay, aun cuando únicamente en parte de esta se localizan cuatros clubes LGBT+.

c) ACT LGBT AC

La Asociación de Comercios y Turismo (ACT LGBT) es una asociación que agrupa negocios LGBT+, no tiene fines de lucro y fue creada en 2013, porque los empresarios no eran escuchados por el gobierno municipal ni por otras instancias gubernamentales, empresariales y sociales, por lo que decidieron agruparse y mostrar una sola cara que buscaría el crecimiento de Puerto Vallarta como destino turístico gay friendly.

d) Colectivo Vallarta LGBT

Esta agrupación no está constituida legalmente; sin embargo, busca la defensa y promoción de los derechos humanos de la diversidad sexual entre los turistas y residentes de Puerto Vallarta. Dentro de su misión destaca la promoción de la salud sexual y el ejercicio ciudadano para lograr la inclusión plena en la sociedad vallartense. 


\section{Análisis de Redes Sociales (ARS) y relaciones de poder que configuran el espacio turístico LGBT+ en Puerto Vallarta}

La configuración socio-territorial de la zona romántica se sostiene con la siguiente clasificación de los actores: a) turistas nacionales e internacionales; b) residentes nacionales y extranjeros; c) asociaciones: asociación de vecinos de la colonia Amapas (Amapas Neighborhood Association) conocido como ANA, ACT LGBT AC, Colectivo Vallarta; d) empresarios: dueños de hoteles, bares, restaurantes, operadoras turísticas, inmobiliarias, entre otros y e) gobierno: Oficina de Igualdad de Género; Secretaría de turismo municipal y presidencia municipal. Todos ellos han logrado la construcción de un espacio turístico configurado como territorio LGBT+ friendly. (ver tabla 1).

Tabla 1. Códigos de actores

\begin{tabular}{|c|c|c|c|c|}
\hline Turistas (T) & Residentes (R) & Empresarios (E) & Asociaciones (A) & Gobierno (G) \\
\hline $\begin{array}{c}\text { T1 } \\
\text { Turista argentino }\end{array}$ & $\begin{array}{c}\text { R1 } \\
\text { Residente mexicana, } \\
\text { heterosexual, } 55 \text { años }\end{array}$ & $\begin{array}{c}\text { E1 } \\
\text { Empresario, dueño } \\
\text { de hotel LGBT+ } \\
\text { friendly }\end{array}$ & $\begin{array}{c}\text { A1 } \\
\text { Asociación de } \\
\text { empresarios, dueños de } \\
\text { negocios LGBT+ }\end{array}$ & $\begin{array}{c}\text { G1 } \\
\text { Oficina de Igualdad de } \\
\text { Género }\end{array}$ \\
\hline $\begin{array}{l}\text { T2 } \\
\text { Turista } \\
\text { inglés }\end{array}$ & $\begin{array}{c}\text { R2 } \\
\text { Residente mexicano, } \\
\text { temporal, gay, } 23 \text { años }\end{array}$ & $\begin{array}{c}\text { E2 } \\
\text { Empresario, } \\
\text { Dueño de cuatro bares } \\
\text { gais }\end{array}$ & $\begin{array}{c}\text { A2 } \\
\text { Asociación activista, } \\
\text { pro derechos humanos } \\
\text { LGBT+ }\end{array}$ & $\begin{array}{c}\text { G2 } \\
\text { Secretaría de turismo } \\
\text { municipal }\end{array}$ \\
\hline $\begin{array}{c}\text { T3 } \\
\text { Turista } \\
\text { canadiense }\end{array}$ & $\begin{array}{c}\mathrm{R} 3 \\
\text { Residente mexicano, } \\
\text { gay, } 30 \text { años }\end{array}$ & $\begin{array}{c}\text { E3 } \\
\text { Empresario, } \\
\text { dueño de hotel gay }\end{array}$ & $\begin{array}{c}\text { A3 } \\
\text { Asociación activista, } \\
\text { dedicada a erradicar VIH }\end{array}$ & $\begin{array}{c}\text { G3 } \\
\text { Presidencia municipal }\end{array}$ \\
\hline
\end{tabular}

\subsection{Análisis de las Relaciones Sociales (ARS)}

Este análisis está planteado para dar cuenta de las relaciones culturales, económicas y políticas que configuran el espacio turístico como LGBT+ friendly, es decir, se realiza a través del modelo de red social y del modelo generado por el capital económico, cultural, político y social que ostentan los actores en la zona romántica y de Amapas en Puerto Vallarta.

La primer forma de análisis de las redes sociales es a través de los lazos o nexos entre las entidades (individuos o instituciones), identificando y analizando los vínculos que se generan para alcanzar un fin, caracterizando cuáles relaciones son más 
fuertes, en tanto a capitales, que permiten tener un papel más sólido en la configuración del espacio turístico LGBT+ friendly.

Los actores con más "conexiones" o posicionados en más caminos tendrán mayores posibilidades de obtener más y mejores recursos a menor costo, además habrá actores que tendrán el potencial de interrumpir, controlar o distorsionar el flujo de recursos entre los demás actores (Faust, 2002, p. 8).

En la figura 2, Red de relaciones de los actores que configuran el espacio turístico vallartense LGBT+ friendly, se presentan los nodos con los 15 actores entrevistados etnográficamente y sus respectivas relaciones señaladas en el entramado. Las relaciones presentadas se basan en las narrativas construidas con las entrevistas y en la observación etnográfica del investigador en reuniones, convenios y espacios de convivencia con los mismos.

\section{Figura 2. Red de relaciones de los actores en Puerto Vallarta LGBT+ friendly}

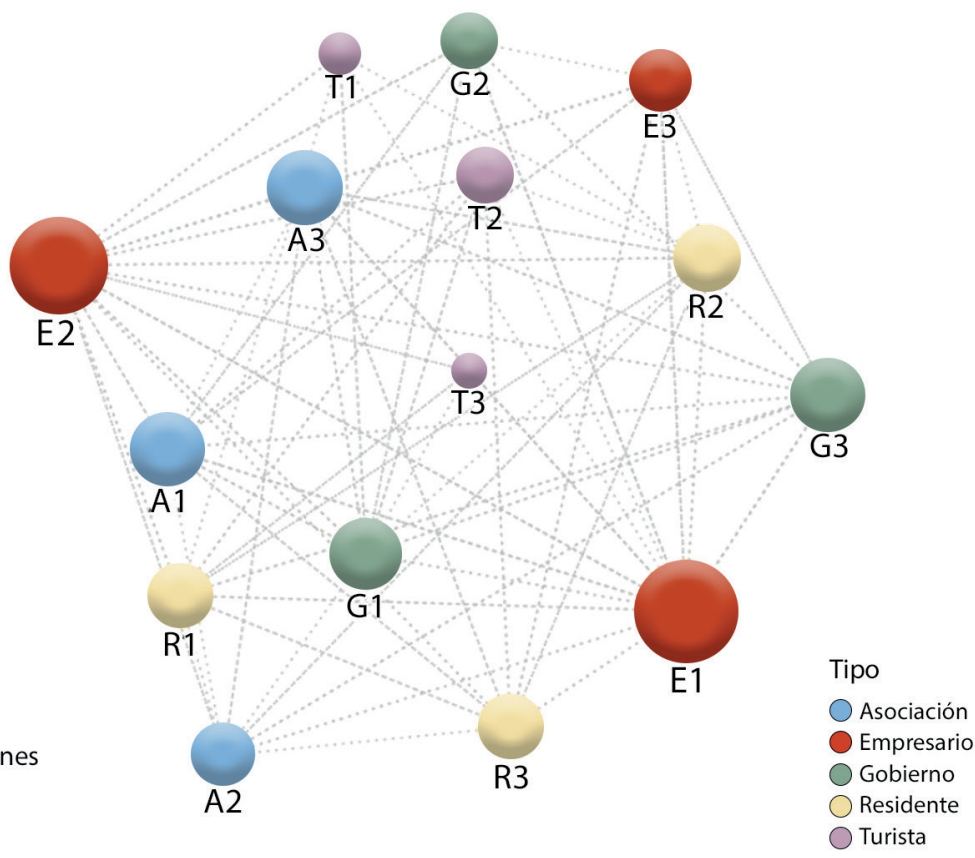

Fuente: Elaboración propia, con base en plataforma onodo.org

En el caso de la red social que se analiza, se tomaron como base dos elementos: una población de actores (turistas, residentes, empresarios, asociaciones y gobierno) y una relación medible, a través de sus contactos o líneas de acción. La forma en que 
se representa la red social es a través de gráficas y matrices como estructuras básicas de información. Como se puede observar en la figura 2, los actores se focalizan en nodos, mientras que sus relaciones se representan con líneas de dirección, arcos y aristas. Esta figura tiene tres funciones en tanto a modelos de redes sociales: 1) servir de modelo representativo de la red; 2 ) mostrar propiedades espaciales de la red y 3) aplicar conceptos de la teoría de grafos para formalizar dichas propiedades (Faust, 2002, p. 5).

En la tabla 2 se presentan los resultados obtenidos de los actores en red; con estos datos y las entrevistas realizadas, el actor turista quedó fuera de este análisis porque se corrobora que estos participan como usuarios del espacio LGBT+. Es importante mencionar que la dinámica de consumo de los turistas se ve moldeada por la forma en la que opera la red.

Tabla 2. Análisis de redes sociales de actores configuradores del espacio turístico LGBT+ friendly

\begin{tabular}{|c|c|c|c|c|c|c|}
\hline Nodo & Tipo & Conexiones & Relevancia & Intermediación & Cercanía & Nuclearidad \\
\hline E1 & Empresario & 30.00 & 1.00 & 6.98 & 0.93 & 12.00 \\
\hline E2 & Empresario & 29.00 & 0.90 & 13.35 & 1.00 & 12.00 \\
\hline $\mathrm{A} 1$ & Asociación & 19.00 & 0.70 & 1.96 & 0.78 & 12.00 \\
\hline G1 & Gobierno & 19.00 & 0.63 & 5.33 & 0.82 & 12.00 \\
\hline G3 & Gobierno & 19.00 & 0.70 & 1.71 & 0.74 & 12.00 \\
\hline $\mathrm{A3}$ & Asociación & 18.00 & 0.68 & 0.88 & 0.74 & 12.00 \\
\hline R3 & Residente & 17.00 & 0.61 & 1.38 & 0.74 & 12.00 \\
\hline R1 & Residente & 16.00 & 0.51 & 3.86 & 0.74 & 12.00 \\
\hline $\mathrm{R} 2$ & Residente & 16.00 & 0.56 & 1.60 & 0.78 & 12.00 \\
\hline E3 & Empresario & 15.00 & 0.60 & 0.59 & 0.70 & 12.00 \\
\hline $\mathrm{A} 2$ & Asociación & 14.00 & 0.54 & 0.52 & 0.74 & 12.00 \\
\hline $\mathrm{G} 2$ & Gobierno & 12.00 & 0.51 & 0.08 & 0.64 & 12.00 \\
\hline $\mathrm{T} 2$ & Turista & 12.00 & 0.44 & 0.61 & 0.67 & 12.00 \\
\hline $\mathrm{T} 1$ & Turista & 7.00 & 0.27 & 0.09 & 0.61 & 7.00 \\
\hline T3 & Turista & 5.00 & 0.19 & 0.09 & 0.56 & 5.00 \\
\hline
\end{tabular}

Fuente: Elaboración propia, realizado en la plataforma onodo.org

En los resultados se observa que los empresarios, en particular E2, el dueño de cuatro bares LGBT+ en la zona romántica, tienen la red de relaciones más amplia, seguido del empresario E1 y en tercer lugar A1, que es la asociación conformada por empresarios, en orden sucesivo estos tres actores sostienen 30, 29 y 19 conexiones 
respectivamente. Dichos actores tienen mayor influencia dentro del grupo y cuentan con mayor capital social, del cual disponen en caso de requerirlo para ejercer el poder en las relaciones (Bourdieu, 1988; 2007; 2015).

Los residentes también mantienen un número significativo de relaciones con los demás actores, debido a que todos ellos viven en la zona romántica y así logran consolidar los lazos sociales con el resto de los actores, aunque en escalas distintas. Por su parte, las asociaciones unidas a los empresarios, logran ser el eje articulador más realzado en la figura, mayor número de conexiones, con 19, 18 y 14 respectivamente, lo cual establece una mayor influencia en las relaciones de poder, su conectividad logra modificar las relaciones sociales de los actores participantes en la zona romántica. De los tres actores de gobierno entrevistados, la presidencia municipal (G3) sostiene mayores lazos sociales establecidos en la red, con 19 conexiones.

La relevancia o fuerza de los vínculos en esta red social recae en E1, E2 y A1, estos actores sostienen los lazos más fuertes a través de las conexiones de entrada y salida, lo cual en términos cuantitativos permite deducir que nuevamente los empresarios poseen un nivel alto de prestigio reconocido por los demás, sumado a que tienen el mayor grado de influencia. Los actores con menor grado de influencia por su nivel de fuerza en los vínculos son los residentes.

El nivel de centralidad de intermediación, es el nivel en que los actores poseen mayor o menor liderazgo, al ejercer mayor control sobre los flujos de comunicación informal (Kuz et al., 2016). En este caso, E2, E1 y G1 demuestran tener mayor liderazgo por el nivel de comunicación que sostienen con los demás actores que configuran el espacio en un territorio LGBT+.

El cuarto elemento que se analizó fue la centralidad de cercanía. En el caso de los nodos con mayor centralidad de cercanía fueron E2 con 1, E1 con .93 y G1 con .82, lo cual significa que aunque los nodos pudieran tener menores conexiones, que en este caso no es así, sus medios les permiten llegar a todos los puntos de la red más rápidamente desde cualquier otro punto, a través de estos nodos con mayor cercanía se puede monitorear el flujo de información de toda la red.

Finalmente, la nuclearidad representa la posibilidad de contacto con otros actores, se observa que la mayoría de los actores tiene 12 posibilidades de unión en sus vértices; lo cual representa que todos ellos tienen alta probabilidad de involucramiento con el resto. Este análisis permite dar cuenta de la estructura en la comunicación 
que se establece en torno a los objetivos de cada actor; no obstante, la capacidad de unión, flexibilidad y de adaptación para (re)configurar el espacio turístico LGBT+ en Puerto Vallarta se complejiza al realizar el análisis cualitativo.

\subsection{Relaciones de poder en Puerto Vallarta}

El marco de la posmodernidad y globalización ha permitido pensar en nuevas realidades sociales, expuesto por las resistencias generadas en comunidades de sentido y de vida que se encuentran en la periferia y en situaciones de exclusión, como la comunidad LGBT+, la cual a través de acontecimientos que desbloquean los flujos de los que está hecho el conflicto promueven puntos de encuentro creativos en lógicas emancipatorias (Useche, 2012). A través de micropoderes se entretejen redes que ponen en juego las subjetividades de los sujetos, que no están representadas en la sociedad dominante y que fueron y siguen en muchos contextos siendo sometidas e invisibilizadas por el orden hegemónico.

Por medio de las entrevistas, reuniones y la experiencia etnográfica vivida en el espacio turístico de la zona romántica durante más de tres años, se constató que los turistas LGBT+, particularmente los gais, son configuradores del espacio turístico por las prácticas sociales que llevan a cabo en el espacio; sin embargo, no son tomadores de decisiones respecto a lo que se hace o no en el contexto turístico.

Los turistas LGBT+ mantienen vínculos con los residentes, empresarios, asociaciones y gobierno por los servicios que les ofrecen; el ejercicio de poder es materializado por el gasto económico que realizan en los diferentes negocios con los empresarios, las aportaciones o donaciones que hacen para las asociaciones, los servicios de información y ayuda de las instituciones de gobierno. El contacto con los residentes es por la convivencia que sostienen al hacer uso del espacio turístico.

Los residentes que forman parte de la comunidad LGBT+, en su mayoría gais que viven en la zona romántica y de Amapas, sostienen vínculos de poder con los demás actores analizados a través de las funciones de agrupación, a través de asociaciones o por ejercer otras funciones, como el ser empresarios del sector turístico, además de que hacen uso del espacio turístico con mayor libertad y/o seguridad al sentirse protegidos por ser una zona amigable con la diversidad sexual.

En este espacio urbano, tanto residentes de la diversidad sexual como heterosexuales, ejercen sus relaciones de poder a través de dos organizaciones que les repre- 
sentan ante los demás actores: la asociación de vecinos de Amapas y la de la colonia Emiliano Zapata (conocida como zona romántica); ambas buscan la armonía del turismo con la funcionalidad y naturaleza del lugar, en otras palabras, que se respete la convivencia de residentes con el sector turístico. Se identificó que los grandes tomadores de decisiones y quienes ejercen la acción sobre las actividades de otros actores son los empresarios, asociaciones e instituciones de gobierno.

Durante los últimos seis años (2015-2021) gobernó el mismo Presidente municipal en Puerto Vallarta, mismo que en apoyo a la comunidad LGBT+ y al turismo desarrolló las siguientes acciones: a) el 20 de abril de 2016 reconoció legalmente el primer enlace matrimonial para parejas del mismo sexo; b) el 6 de abril de 2017 se inauguró la Oficina de Igualdad de Género, atención y orientación turística a la diversidad social, en el parque Lázaro Cárdenas de la zona romántica y c) 25 de mayo de 2017 fue el primer alcalde en participar en una marcha del orgullo gay en Puerto Vallarta (Andrade, 2016; Beltrán, 2017; Moguel, 2017).

El Alcalde reconoció la importancia que tienen los empresarios en la construcción del espacio LGBT+ al afirmar en una reunión de empresarios organizada ACT LGBT lo siguiente:

Aquí, ustedes los empresarios, ¡ustedes son los que dirigen a los funcionarios públicos!... se les va seguir apoyando, impulsando las actividades y yo en lo que pueda con mucho gusto, yo estaré ahí para ayudarlos (Dávalos y Sánchez, Presidente municipal de Puerto Vallarta y Presidente de ACT LGBT AC, entrevista etnográfica, 9 de febrero de 2018).

El Presidente municipal se comprometió a seguir apoyando a la comunidad LGBT+ hasta el final de su mandato e incluso reiteraba su apoyo en caso de ganar las elecciones en julio de 2018, lo cual sucedió. La asociación manifestó su agradecimiento y a través de su líder, Armando Sánchez, demandó:

... lo principal sería la cuestión de votaciones en el Fideicomiso de Turismo y qué se hace en abogar por la comunidad... donde nosotros siempre hemos dicho que se necesita más fuerza para que se reconozca a la comunidad (LGBT+) como tal, entonces para poder ver campañas de antidiscriminación o de conciliación, de respeto, de igualdad, etc., ¡hay muchas cosas que no vemos en ninguna campaña! y que por el destino que es, que es Puerto Vallarta y que tiene la comunidad gay, mucha diversidad, localmente no vemos muchas campa- 
ñas que eduquen a las generaciones que vienen sobre este tipo de situaciones (Dávalos y Sánchez, Presidente municipal de Puerto Vallarta y Presidente de ACT LGBT AC, entrevista etnográfica, 9 de febrero de 2018).

La respuesta del edil siempre fue positiva e insistió en que fue el primer gobernante del municipio de Puerto Vallarta en atender las peticiones de la comunidad LGBT+, incluso comentó a los representantes de ACT LGBT, que fue el primero en desfilar en el Vallarta Pride 2017, hecho que fue catalogado como histórico, pues en las pasadas administraciones municipales, ningún alcalde había participado en los desfiles del orgullo gay en esa localidad, para manifestar su apoyo para la comunidad LGBT+.

Los empresarios tienen como base el poder económico, privilegio que les permite diferenciarse en las relaciones de poder. Sus efectos al agruparse en la asociación ACT LGBT se vieron potenciados al conjuntar el capital económico con el cultural que posee cada uno de ellos. Su objetivo, además de generar mayores riquezas derivado del usufructo del espacio turístico, es hacer valer y respetar los derechos humanos de la comunidad LGBT+, que son sus principales clientes en la zona romántica y de Amapas.

Como se observa en el caso de la red de actores establecida dentro del campo del turismo LGBT+ en Puerto Vallarta, esta depende de dos mecanismos básicos para ejercer el control sobre otros: el primero se basa en la capacidad de generar la red con objetivos específicos, como generar mayor derrama económica derivado del gasto de turistas y residentes LGBT+, respaldados con el argumento de la no discriminación y de aceptación social para la comunidad diversa. El segundo tiene que ver con la capacidad de conectar diferentes redes y actores que comparten objetivos y combinan capitales (económicos, culturales y sociales), como lo hace la sociedad civil a través de asociaciones, los empresarios y los turistas y residentes que demandan no solo la infraestructura para realizar sus prácticas, sino la conformación de un espacio en el cual puedan ser y hacer lo que en otros lugares no se les permite por su orientación sexual o identidad de género.

Lo común de la red son las ideas, visiones, marcos y proyectos que tienen como eje las identidades de esta diversa comunidad, tal es el caso reciente de la unión creada entre residentes, empresarios y asociaciones de orden civil e instancias del gobierno municipal que colaboran para el desarrollo de la comunidad LGBT+ en Puerto Vallarta, en la búsqueda y concreción de dos proyectos: 1) la aprobación del reglamento para prevenir y eliminar la discriminación en el municipio costero, que 
fue aceptado y publicado en agosto de 2018 y 2) el hermanamiento con la ciudad de San Francisco, formalizado en septiembre de 2018. Ambos proyectos buscan proyectar y consolidar la presencia y fuerza de la comunidad LGBT+ en este destino turístico y no únicamente en territorio de la zona romántica.

El reglamento fue gestionado principalmente por una asociación de orden civil formado por residentes LGBT+ de Puerto Vallarta denominado Colectivo Vallarta LGBT desde el año 2016, el cual fue impulsado por la comunidad de empresarios y otras asociaciones que apoyan a la misma comunidad diversa, quienes con la suma de sus capitales y gracias a su posición en la estructura social ejercieron su poder para lograr su aprobación. El director de la referida agrupación señala al respecto:

El Colectivo Vallarta LGBT tiene como fin [apoyar] los Derechos LGBT+ de la población de Puerto Vallarta, buscamos que la comunidad LGBT+ tenga los mismos derechos humanos que el resto de la población en México y el mundo, buscamos dejar claro que todos somos iguales. Somos personas, tenemos los mismos derechos, buscamos el derecho al matrimonio igualitario, buscamos el derecho a la adopción, buscamos todos los derechos de las personas, el derecho de las personas trans, de cambio de nombre y eso es lo que se busca, el cambio de los derechos, que todos, que todos tengamos los mismos derechos que no haya exclusión de derechos de nadie (Ramírez, Director de Colectivo Vallarta LGBT, entrevista etnográfica, 17 de noviembre de 2017).

Esta estrategia expuesta por Colectivo Vallarta LGBT, en conjunto con otros representantes de la sociedad, resultaría en una victoria mutua: para la asociación se firmaría un reglamento que estaba en revisión por más de tres años, mientras que el gobierno municipal lograría el hermanamiento de ambas ciudades en septiembre del mismo año. Como afirmaría Foucault (1979), no hay relaciones de poder sin resistencias basadas en estrategias.

Una vez aprobado el Reglamento, sirvió como fundamento legal y soporte para formalizar el hermanamiento con la ciudad de San Francisco, California, la cual es considerada como la ciudad ícono y modelo de la comunidad gay en el mundo. Este proyecto fue apoyado por los mismos empresarios, asociaciones y residentes vallartenses que buscan generar un sentido de mejoría en su situación, no discriminación y respeto de derechos humanos de la comunidad LGBT+ en Puerto Vallarta, así como su aceptación social. 
La acción en colectivo de los diferentes actores que ponen en juego sus capitales (económicos, culturales, sociales y simbólicos) logran introducir nuevas instrucciones y códigos en la programación de la red (Castells, 2009), que en el caso de la comunidad LGBT+ en Puerto Vallarta consiguió la aprobación de reglamentos que garantizan el respeto de las minorías y permitieron la creación de acuerdos para seguir operando como red con un mayor grado de visibilización, presentando resistencias al poder mediante la colaboración con esos mismos poderes.

La dominación y la resistencia a la misma en el caso de la comunidad LGBT+ en Puerto Vallarta han cambiado de carácter, adecuándose a la estructura social que se va modificando, permitiendo gobernar al poder y resistir como contrapoder en forma de red, procesando sus acciones que resultan contradictorias en algunas ocasiones pero que logran influir en la toma de decisiones que les atañen, mientras que otros integrantes de la comunidad resisten mediante el activismo de protesta. Finalmente, se afirma que entender las relaciones de poder de los diferentes actores que configuran espacios-territorios turísticos, es prioritario, ya que estas relaciones se encuentran entramadas en articulaciones entre lo global y lo local.

\section{Conclusiones}

El trabajo con este andamiaje teórico-metodológico permite aseverar que el espacio turístico es construido y configurado por una serie de relaciones e interacciones entre diversos actores que desarrollan prácticas materiales y simbólicas mediadas por la política, la economía y la cultura inscrita en el territorio.

Se afirma entonces que la configuración de un espacio turístico amigable con la diversidad sexual ha modificado las coordenadas espaciotemporales a través de prácticas sociales que realizan los turistas LGBT+, así como actores que las posibilitan en red. Estos actores colaboran con discursos y acciones en la construcción de un sentido social de respeto e inclusión de la diversidad sexual, en un territorio en el que cotidianamente conviven residentes y turistas.

Esta configuración del territorio abre la oportunidad a prácticas turísticas de la comunidad LGBT+; todo esto como resultado de un proceso de negociación interno, es decir, involucrar a los actores (gobierno, empresarios, asociaciones civiles) y a los turistas en su calidad de consumidores, bajo un contexto nacional e internacional mediado por instancias que inciden en políticas públicas de México. 
Es importante enfatizar que un espacio turístico, en tanto espacio configurado, no se asimila en su totalidad al destino turístico, es más una delimitación parcial de su territorio. La clave se encuentra en el sentido de valorización, pertenencia y (re)significación de la comunidad residente y visitante con respecto a las prácticas que se llevan a cabo en el mismo. Es decir, un espacio turístico formaría parte del destino turístico junto con otros espacios turísticos configurados y apropiados por los sujetos que lo viven y (re)configuran. En el caso de Puerto Vallarta, la zona romántica y de Amapas conforman lo que se reconoce como espacio turístico LGBT+.

Si bien no se puede sostener con los datos empíricos analizados en esta investigación que el caso estudiado sea un gueto construido por políticas públicas o por consentimiento social de sus residentes y turistas, sí se puede sostener la idea de que este espacio turístico puede ser resultado de un proceso de aislamiento o "guetización" que permite habilitar y desarrollar ciertas prácticas que en otros espacios del destino turístico no son habilitadas ni legitimadas.

El Gobierno Federal en México reconoce la existencia de ocho destinos turísticos que se promueven como destinos amigables con la comunidad LGBT+; no obstante, se sostiene que en el caso de Puerto Vallarta no es todo el destino LGBT+, sino un territorio específico que habilita y legitima prácticas concretas asociadas a la diversidad sexual, vinculándolas con productos turísticos específicos.

A manera de cierre, se sostiene que la dinámica de las redes de poder al interior del campo del turismo proviene del acto de sumar capitales para ejercer mayor presión y alcanzar los objetivos perseguidos por cada actor de manera legítima, a la par del uso de múltiples mecanismos (marchas, denuncias y/o acuerdos gestionados previamente por cabildo, por ejemplo) para alcanzarlos. Si bien estos mecanismos no son excluyentes entre sí, cabe hacer mención que los acuerdos que se logran en cabildo, es decir, en reunión de la mayoría de los actores, incluyendo a las instituciones gubernamentales, son los que logran verse materializados más rápidamente en acciones y políticas públicas que impactan en la configuración de los espacios turísticos. 


\section{Referencias}

Andrade, C. (2016, 12 de febrero). Se registra la primera pareja para matrimonio igualitario. Tribuna de la bahía. https://bit.ly/3wnA6tc

Benach, N. (2016). ¿Ciudades en el mapa o en la guía turística? Venta de la ciudad y sentidos del lugar. CIDOB d'Afers Internacionals, 113, 89-105. https://doi. org/10.24241/rcai.2016.113.2.89

Beltrán, B. (2017, 6 de abril). Inauguran oficina de Igualdad de Género en PV. Tribuna de la bahía. https://bit.ly/3hi70XV

Beltrán, B. (2018, 27 de octubre). Firma Colectivo Vallarta LGBT convenio de colaboración con la CEDHJ. Tribuna de la bahía. https://bit.ly/36gDSda

Borja, J. (2013). Revolución urbana y derechos ciudadanos. Alianza.

Bourdieu, P. (1984). Espacio social y génesis de las "clases". En P. Bourdieu (Ed.) Sociología y cultura (pp. 281-309). Consejo Nacional para la Cultura y las Artes - Grijalbo.

Bourdieu, P. (1988). La distinción. Altea Taurus - Alfaguara.

Bourdieu, P. (1996). Espacio social y poder simbólico. En P. Bourdieu (Ed.), Cosas dichas (pp. 127 - 142). Gedisa, Barcelona.

Bourdieu, P. (1997). Razones prácticas. Anagrama.

Bourdieu, P. (2007). El sentido práctico. Siglo XXI.

Bourdieu, P. (2015). La dominación masculina. Anagrama.

Bronfman, M. (2000). Como se vive se muere. Familia, redes sociales y muerte infantil. Universidad Nacional Autónoma de México.

Castells, M. (2009). Comunicación y poder. Alianza editorial.

Collignon, M. (2011). Discursos sociales sobre la sexualidad: narrativas sobre la diversidad sexual y prácticas de resistencia. Comunicación y Sociedad, 16, 133160. https://bit.ly/3AvdUAJ

Cortés, F., Escobar, A. y González, M. (2008). Método científico y política social: a propósito de las evaluaciones cualitativas de programas sociales. El Colegio de México. 
Daniilidis, A. y Beaumont, J. (2015). Socio-spatiality, power relations and the right to the city. http://dx.doi.org/10.13140/RG.2.1.3946.8881

Faust, K. (2002). Las redes sociales en las ciencias sociales y en el comportamiento. En J. Gil y S. Schmidt (Eds.), Análisis de redes sociales. Aplicaciones en ciencias sociales (pp. 1-14). Universidad Nacional Autónoma de México.

Foucault, M. (1979). Microfísica del poder (J. Varela y F. Álvarez-Uría, Trads.). Edissa. https://bit.ly/3hFRAM0

Foucault, M. (1988). El sujeto y el poder. Revista Mexicana de Sociología, 50(3), 320. https://doi.org/10.2307/3540551

Giddens, A. (2006). La constitución de la sociedad. Bases para la teoría de la estructuración (J. L. Etcheverry, Trad.). Amorrortu Editores.

Giménez, G. (2005). Territorio e identidad. Breve introducción a la geografía cultural. Trayectorias, VII(17), 8-24. https://bit.ly/2SSal17

Giménez, G. (2007). Estudios sobre la cultura y las identidades sociales. Instituto Tecnológico y de Estudios Superiores de Occidente.

Guber, R. (2015). Método, campo y reflexividad. Siglo XXI Editores.

Hage, P. y Harary, F. (2002). ¿Qué es un hipercubo? Un código binario para las relaciones de parentesco. En Gil y Schmidt (Eds.), Análisis de redes sociales. Aplicaciones en ciencias sociales (pp. 15-22). Universidad Nacional Autónoma de México.

Harvey, D. (2008). El derecho a la ciudad. New Left Review, (53), 23-39. https://bit. ly/3yuzALB

International Gay \& Lesbian Travel Association (IGLTA). (2017, 28 de octubre). Acerca de. https://bit.ly/3jKAoYl

Kuz, A., Falco, M. y Giandini, R. (2016). Análisis de redes sociales: un caso práctico. Computación y Sistemas, 20(1), 89-106. https://doi.org/10.13053/cys-20-12321

Moguel, L. (2017, 6 de abril ). Puerto Vallarta construye una sociedad incluyente. Noticias PV. https://bit.ly/2SNxXt4

Organización Mundial del Turismo (OMT). (2017). Second global report on LGBT tourism. https://bit.ly/3ytG2IN 
Out Now Global. (2015, 18 de marzo). New research: LGBT travel - Where will gays and lesbians holiday in 2015. https://bit.ly/3AuUwUi

Saarinen, J. y Wall-Reinius, S. (2019). Enclaves in tourism: producing and governing exclusive spaces for tourism. Tourism Geographies, 21(5), 739-748. https:// doi.org/10.1080/14616688.2019.1668051

Secretaría de Turismo de México (Sectur). (2016, 16 de mayo). Datatur Jalisco. https://bit.ly/3wjLBBO

Secretaría de Turismo de México (Sectur). (2017a, 30 de enero). Fortalece Sectur Turismo LGBT para hacer de México un destino de vanguardia. https://bit.ly/3wjLOF6

Secretaría de Turismo de México (Sectur). (2017b, 21 de marzo). Sectur impulsa estrategias de diversificación para turismo LGBT. https://bit.ly/3xn51XO

Secretaría de Turismo de México (Sectur). (2018, 17 de mayo). Turismo LGBT. https:// bit.ly/3yp6Fs3

Senado de la República. (2014, 14 de abril). Propuesta para crear Subsecretaría de la Diversidad Sexual en México. https://bit.ly/3hgAYvf

Sosa, M. (2012). ¿Cómo entender el territorio? Editorial Cara Parens.

Tashakkori, A. y Teddlie, Ch. (1998). Mixed methodology. Combining qualitative and quantitative approaches. Sage Publications.

Useche, O. (2012). Diferencia, subjetividades en resistencia y micropolítica del acontecimiento. En C. Piedrahita, A. Díaz y P. Vommaro (Comps.), Subjetividades políticas: desafíos y debates latinoamericanos (pp. 95-109). https://repositorio. idep.edu.co/handle/001/2175 